\title{
Red single-photon emission from InAs/AIGaAs quantum dots
}

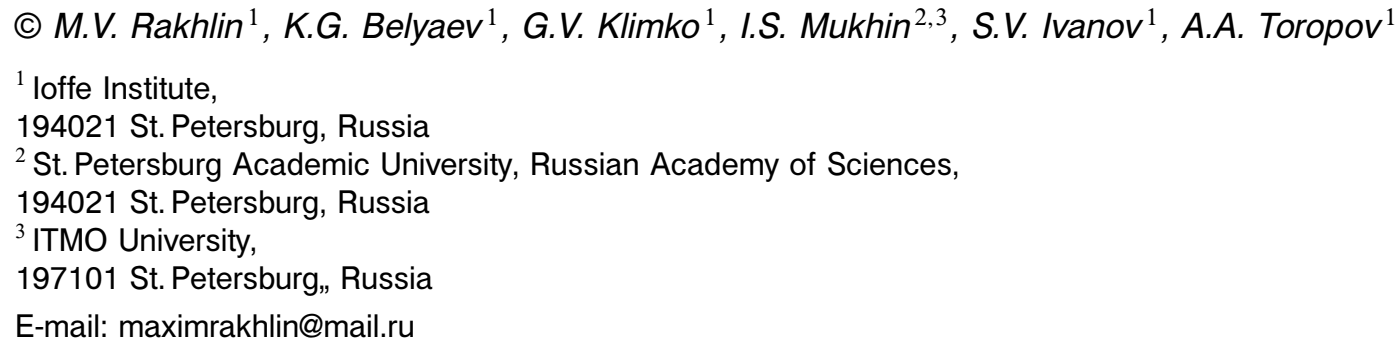

We report on single-photon emission of InAs/AlGaAs self-assembled quantum dots (QDs) grown by molecular beam epitaxy. By varying the growth conditions the QDs luminescence could be tuned over a wide wavelength range from 0.64 to $1 \mu \mathrm{m}$, including red part of the visible spectrum. Emission properties of individual QDs are investigated by micro-photoluminescence ( $\mu$-PL) spectroscopy using $500 \mathrm{~nm}$-size etched mesa structures. Autocorrelation functions of photons from single QDs, measured in the wide spectral range demonstrate antibunching effect at zero delay time with a value of $g^{(2)}(0) \sim 0.17$ that is a clear evidence of non-classical light.

\section{Acknowledgement}

The authors gratefully acknowledge the financial support of the Russian Science Foundation (project \#14-22-00107). 